\title{
COLLECTIVE WISDOM OF FLIPPED CLASSROOMS IN HONG KONG HIGHER EDUCATION: PREPARING FOR POTENTIAL CHALLENGES
}

\author{
Paul Lam, Carmen K. M. Lau and Chi Him Chan \\ The Chinese University of Hong Kong, Hong Kong
}

\begin{abstract}
The flipped classroom approach is widely regarded to be able to enhance teaching and learning. Despite its many advantages, it is still in the early stage of implementing in Hong Kong. This may be due to the potential challenges such as learners' limited participation and adaptation problems. The purpose of this article is to discuss the challenges based on Brame's (2013) four key elements of the flipped classroom - Exposure, Incentive, Assess and Activities. Particularly, we put emphasis on how teachers from Hong Kong higher education address the four aspects of challenges in their flipped classroom planning and design. Twenty-six teachers from five universities in Hong Kong were invited to complete a teaching profile for their coming flipped courses which they were asked to elaborate their teaching goals and how they would execute the course. The teaching profiles revealed that the teachers devised a wealth of different strategies in implementing flipped classroom. We generalized their careful planning into eleven tips that can be seen as the collective wisdom of the teachers, as well as potential solutions to the flipped classroom challenges. This article summarizes the first phase of our study that eventually will examine the success factors of the flipped classroom approach in Hong Kong higher education.
\end{abstract}

\section{KEYWORDS}

Flipped Classrooms, Challenges, Tips

\section{INTRODUCTION}

The flipped classroom is an idea to reverse the traditional learning environment by moving lectures out of the classroom and bringing activities such as projects, discussions, and presentations into the classroom, hence it is called "flipped" or "inverted" classroom. Unilateral information presenting activities such as traditional lectures are removed from the classroom, so that classroom time is freed for activities that focus on assimilating or applying knowledge. The content and form of delivery of both in-class and out-of-class activities can be varied, hence there is no single model of flipping the classroom (Sams, 2011). For example, lectures can be delivered through distance learning technologies such as multimedia or online delivery, and activities of different teaching styles or the use of active learning strategies that focus more on application of knowledge can be applied in classroom time according to teaching needs (Baker, 2000; Lage et al., 2000). This approach has two major advantages: it promotes active learning in a constructive learning environment and increases interaction.

A constructive learning environment is a learning environment that allows students to construct their knowledge through active discovery. The flipped classroom approach creates such a learning environment by combining video lectures and hands-on classroom experience from different in-class activities. Students can construct their knowledge by actively exploring different learning materials and participating in different activities (Gannod et al., 2008). The lecture materials in a flipped classroom are delivered asynchronously out of class, so students can access the material whenever they need, meaning that they can control their own learning progress (Gannod et al., 2008). This provides a higher degree of flexibility and a greater sense of responsibility for their learning (Baker, 2000; Lage et al., 2000). Different kinds of learning activities are introduced into the classroom to engage students' involvement. This also agrees with the principle of active learning (Gannod et al., 2008). 
Interaction is also increased in the flipped classroom. Teachers have a greater chance to engage their students, which is more rewarding than repeating lectures. On the other side of the coin, students receive immediate feedback from teachers. Particularly, weaker students receive more attention, since their teacher works with them directly (Gannod et al., 2008). Moreover, interaction among students also increases because discussions are encouraged. Through explaining concepts to each other, their understanding is reinforced, knowledge is consolidated, and they can learn from their peers (Baker, 2000; Gannod et al., 2008).

According to Gökçe Akçayır and Murat Akçayır (2018), the number of research articles on the flipped classroom steadily increased after 2012, with 79\% of those studies conducted between 2015 and 2016. With growing research interest and efforts in the pedagogy, various challenges on its implementation were discovered. One of the most frequent challenges discovered was limited student preparation before class. Other challenges included students' adaptation problems caused by their anxiousness and resistance, teachers' unwillingness to participate and a lack of school support or technological support (Akçayır and Akçayır, 2018).

This article examines and categorizes the abovementioned challenges according to a framework based on four key elements suggested by Brame (2013). In addition, we explore how university teachers in Hong Kong designed their flipped classrooms in response to the four areas of challenges through a cross-university study conducted in 2018. Twenty-six teachers from five universities in Hong Kong were invited to complete a teaching profile on how they would design their flipped courses. By analyzing these teaching profiles, we discovered eleven tips on the level of course design which might be able to meet the challenges.

\section{CHALlengeS}

In her guide to flipping the classroom, Brame identifies four key elements in the flipped classroom, namely "exposure", "incentive", "assess" and "activities". These key elements should be well thought out before course delivery to guarantee its success. In a successful flipped classroom, "incentive" motivates students to gain knowledge through accessing "exposure" material before class while teachers evaluate students' understanding of the material through "assess", and tailor in-class "activities" according to students" need to foster their assimilation of the knowledge. However, different challenges may occur in these four key elements and hamper the success of a flipped classroom.

\subsection{Exposure}

"Exposure" implies the opportunity for students to gain first exposure prior to class. In a flipped classroom, learning materials are provided for students to study before class so that they obtain basic knowledge and skills to participate in high cognitive activities during class. (Brame, 2013).

Although Brame suggests that pre-class exposure does not need to be high-tech, most teachers still choose to deliver learning materials in the form of videos (Akçayır and Akçayır, 2018). But producing a video poses a frequent challenge - how to ensure its quality? Teachers are reported to be hesitant to use videos provided by others and do not have the skills or resources to produce good quality videos. As a result, students' understanding of the contents is undermined by tedious and dry videos or videos with poor audio quality. On the other hand, the length of a video also affects students' view counts. The longer the video, the fewer the view counts (Akçayır and Akçayır, 2018). The form and medium of delivering pre-class exposure play an important role in students' perception of the teaching approach.

Besides the form and medium, additional assistance is needed for pre-class exposure. Some students report that they are unable to get help, for they cannot ask questions immediately when they are viewing pre-class materials (Fautch, 2015; Chen et al., 2015). It is also suggested that clear guidelines or instruction are crucial for pre-class learning (Wanner and Palmer, 2015).

\subsection{Incentive}

"Incentive" is a "pull" factor that motivates students to participate in the flipped classroom learning processes. Comparing to the traditional classroom, the workload of students in a flipped classroom increased because flipped classroom requires students to prepare for the class and participate in class actively (Khanova et al., 
2015). Therefore, some additional incentives would be crucial for motivating students to participate in the flipped classroom learning process.

There are two types of incentive for learning. Extrinsic incentive refers to individuals engaging in learning because it leads to separable outcomes, in which a score is an example. Intrinsic incentive refers to individuals engaging in learning because it is inherently interesting and enjoyable (Abeysekera and Dawson, 2014). Brame suggests that tasks should be set to indicate that students have finished their preparation before they attend class, and extrinsic incentive can be provided by assigning scores to motivate students to finish these tasks (Brame, 2013). Apart from the extrinsic incentive of giving scores, intrinsic incentive such as promoting students' acceptance of the flipped classroom approach is also an important part in the "incentive" element. Previous studies show that some students do not participate in the flipped classroom because they are anxious about the flipped classroom approach, they are uncertain about what the flipped classroom may entail. Some students do not view it as useful comparing to traditional lectures (Akçayır and Akçayır, 2018). Moreover, the flipped classroom approach may disfavor students who are less active (Strayer, 2007). They may find themselves in an unsafe situation where they need to participate actively in class instead of passively listening to lectures as in the traditional lecturing approach (Chen et al., 2014). Worse still, they may even think that the flipped learning environment is unfair or unbearable. All of these factors contribute to students' resistance to the flipped classroom approach (Akçayır and Akçayır, 2018).

\subsection{Assess}

"Assess" is a mechanism to assess students' understanding. The assessment on pre-class exposure aims mainly at assessing students' understanding. Teachers can identify areas that students are struggling with so that teachers can tailor in-class activities according to students' needs. These assessments should be graded for completion rather than effort so that they can also serve as evidence of students' preparation. (Brame, 2013).

Challenges usually occur in using the assessment to check students' preparation. Although some teachers assign quizzes at the beginning of a class, it is still difficult to ensure that students study the pre-class materials thoroughly. It is reported that formative assessment that graded upon completion is difficult to hold students accountable for their preparation (Fautch, 2015), both because the questions are not hard enough and because the grade is insignificant. In addition, online quizzes are vulnerable to students' inappropriate behaviors. Students might guess all the choices of a multiple-choice question until getting the right answer or search through the material to find correct answers just to complete the assessments (Koh et al., 2018). Students might exploit the advantage to finish the assessments without learning from the materials.

\subsection{Activities}

The "Activities" component means in-class activities. Students learn basic knowledge outside the classroom, therefore different activities can be introduced into class time to promote deeper learning and improve their skills. Brame provides a board range of activities and advises teachers to make their choice according to the learning objective of their class and the culture of their discipline (Brame, 2013).

Planning activities can be challenging for teachers. If teachers fail to plan an appropriate number of types of activities and guide students through these activities, students may withdraw from the class. Strayer (2007) provides an example for this. In Strayer's class, too many types of activities with different goals were given to his students. They felt that it was difficult for them to meet the expectations of each activity and to navigate the expectation for the whole class, and therefore they "were "lost' by the end" (Strayer, 2007, p. 160).

Another challenge for teachers is the need to make more effort in catching students' attention to in-class activities. The class would be disrupted if students work on tasks unrelated to the class instead of participating in in-class activities (Strayer, 2007). This challenge is not only on teacher's ability to control the classroom but also on designing engaging in-class activities.

To conclude, various challenges may arise in these key elements, including the need of good quality videos and additional assistance for students' exposure; the need of incentive, both extrinsic and intrinsic, to motivate students; the need for assessment as a means to ensure students' preparation; and the adequate numbers of types of activities and their ability to engage students. These challenges can hamper the 
successful implementation of a flipped classroom. However, these challenges can be met by careful planning of the course. By examining the course design of teachers from Hong Kong higher education institutions, this article tries to extract some tips that may be able to meet these potential challenges.

\section{METHOD}

\subsection{Participants}

Five universities (the Chinese University of Hong Kong, City University of Hong Kong, the Education University of Hong Kong, Hong Kong Baptist University, and the Hong Kong Polytechnic University) are engaged in a collaborative project (https://www.flippedclasshk.net/) to support teachers to use the innovative pedagogy. The project invited teachers who intended to implement the flipped classroom approach in their courses to participate in the first phase of the project. Twenty-six teachers from various fields of study were recruited. The courses that they intended to flip were also offered to students of different levels. Demographics of the courses can be seen in the table below:

Table 1. Demography of the Courses Participated

\begin{tabular}{|l|l|l|l|}
\hline Level & No. & Field of Study & No. \\
\hline Courses for undergraduates & 23 & Arts & 1 \\
\hline \multirow{3}{*}{ Courses for graduates } & 3 & Business and & \\
\hline & & Administration & 5 \\
\hline & & Education & 2 \\
\hline & & Engineering & 2 \\
\hline & & Textile & 1 \\
\hline & Law & 1 \\
\hline & & Nursing & 2 \\
\hline & & Science & 8 \\
\hline Total No. of Courses & Social Science & 4 \\
\hline & 26 & & 26 \\
\hline
\end{tabular}

\subsection{Procedures}

The teachers were asked to complete a teaching profile before the semester began. The teaching profile was divided into two parts, the first on basic information of the course, and the second on course design. A sample of the teaching profile is attached in the appendix.

Basic information of the course included course name and code, its date, time, venue and estimated number of students. This information helped us identify the size of each class, the level of targeted students and the field of study of each course.

Questions on the course design are closely related to Brame's framework. Teachers were asked why they chose to implement the flipped classroom approach in their course and what goals they aimed to achieve. There were also questions about activities before class (exposure), ways to encourage students to complete the activities (incentive), activities during class and activities after class (activities), and assessment design (assess). Some teachers also wrote down their concerns regarding related issues in their teaching profile. The course design ideas collected in this part provided the data which is the collective wisdom of our teachers in implementing the innovative pedagogy.

\section{RESULTS}

From the teaching profiles, we can see that the teachers devised different measures to avoid the failure of their flipped classes. Through examining these measures, we extracted and generalized eleven tips that may 
be useful in meeting the challenges stated above. These tips are organized according to the four key elements and presented as follows.

\subsection{Exposure}

\subsubsection{Ensuring Video Quality and Using a Variety of Media}

Videos were still the most common type of medium in delivering pre-class exposure as seen from the teaching profiles. For some teachers, however, making good quality videos remained a challenge. Two teachers explicitly stated that they would incorporate existing videos provided by others into their courses. Existing video lectures, such as Khan Academy or TED talks, are proven to be useful in minimizing boredom and distraction (Gilboy et al., 2015). Many publishers have also developed nice recorded videos (McGivney-Burelle and Xue, 2013). The quality of the video can be ensured by using these videos. Besides, teacher's burden on producing material might also be reduced.

We found that the teachers also employed a variety of media to deliver pre-class exposure in order to enrich students' learning environment. Pre-class readings and documents were assigned to students not only in text form but also as digital documents or websites. Some courses required students to search for texts they needed in the web by themselves. One course stressed the use of various formats in delivering pre-class exposure, including text, video, audio, and multimedia. Other types of pre-class exposure included animation, VR trip to a specific site, or visits to the showroom of famous brands. As stated, the combination of different learning materials and learning activities can provide a constructive learning environment for students (Gannod et al., 2008). In addition, the wide array of media and forms would also be able to stimulate students' interest.

\subsubsection{Offering Additional Assistance to Students}

A persistent problem in the flipped classroom approach is that students cannot receive immediate responses when they have questions on the pre-class exposure materials (Fautch, 2015; Chen et al., 2015). Previous flipped classes encourage students to write down their questions and save them for lectures (Fautch, 2015). With the help of online discussion forums, students can raise questions whenever they needed help, and teachers can offer instant feedback and immediate assistance to students by text message (Fautch, 2015). Most of the teachers in our project utilized the online discussion forum in the learning management software provided by their own universities, such as FutureLearn, Moodle and Canvas. One course even provided a discussion forum that allowed students to discuss with previous students of the course. These discussion forums enabled students to raise questions or discuss with each other even when they were out of class.

Besides, guiding questions would be useful to enhance students' understanding of the materials. Guiding question, if well-designed, can help students review learning contents, stimulate critical thinking, thus having a positive effect on learning performance (Thai et al., 2017). Well-designed guiding questions can guide students to understand the pre-class exposure materials, thus reducing students' questions. Two courses provided guiding questions with some text-based materials to students as pre-class exposure, and both courses had discussions on pre-class exposure material as their in-class activities.

\subsection{Incentive}

\subsubsection{Giving Score as an Incentive}

Incentives are necessary to motivate students to participate in a flipped classroom. A score is a strong extrinsic incentive for students (Brame, 2013). Most teachers chose to score the tasks relating to pre-class exposure for the final grade of their course. In fourteen courses that provided a detailed assessment scheme, eleven indicated that some score between $5 \%$ and $20 \%$ of final grade were assigned to tasks related to pre-class exposure. The other three courses listed the tasks relating to pre-class exposure as parts of "continuous assessment" that occupied between $30 \%$ and $50 \%$ of the final grade. In addition to directly assigning grades to tasks, teachers also incorporated the content of pre-class exposure material into mid-term and final examination. One teacher decided to give bonus scores as an incentive, but no details on the arrangement of bonus marks were provided. Teachers believed that score is a common incentive for students to participate in any learning activities. 


\subsubsection{Increasing Students' Acceptance of the Flipped Classroom}

Students' resistance to the flipped classroom approach posed a great challenge to their incentive, therefore, it is crucial to reduce their resistance. The teachers in our project try to increase students' acceptance by explaining the advantages of the flipped classroom approach. Three teachers stimulated students' incentive by explaining the advantages of the flipped classroom and the usefulness of pre-class preparation to students. They explained to students that students can learn at their own pace, thus enhancing their autonomy. In a biology course, the teacher explained to his students that both understandings of the concepts and adequate training on the laboratory skills were important for experiment results, so students must acquire knowledge by watching pre-class exposure before class and do experiments in class time. For courses that had discussion activities, teachers explained to students that it would be easier for them to engage in in-class discussions with preparation before class. It is believed that if students realize that the flipped classroom can satisfy their needs for autonomy, their intrinsic incentive in learning can be facilitated (Abeysekera and Dawson, 2014).

\subsection{Assess}

Assessment in the flipped classroom should aim at assessing students' understanding. The assessment result can demonstrate students' weakness, and teachers can tailor their in-class activities to focus on the students' need. This can further improve students' understanding. However, the teachers in our project seldom explored this aim of assessment. Instead, they emphasized the use of different assessment tasks to check students' preparation. This might be because they regard the need to ensure students' preparation as one of the most serious challenges.

\subsubsection{Assigning Pre-class Tasks}

Regarding the difficulty of ensuring students preparation, most courses required students to finish a variety of tasks before class. While those tasks mainly included multiple choice questions and online quizzes, they were designed to ensure that students had studied the material thoroughly. For example, questions on pre-class exposure were made to cover more contents of the materials. In addition, some courses required students to finish worksheets, write summaries, submit comments, or raise discussion questions as pre-class tasks. These tasks reduce the possibility that students exploit the advantage to finish the task without studying the pre-class exposure materials.

\subsubsection{Assessing Students through In-Class Activities}

Different kinds of in-class activities can also help check students' preparation before class. Four teachers stated that they would ask questions in class occasionally to check if students had prepared for class. Besides, students were required to finish tests or online quizzes in class. Six courses set quizzes in their classes by using different kinds of student response systems. These assessments were designed to be based on pre-class exposure. In-class discussions were also arranged on the basis of pre-class exposure. Students' preparation could be checked by observing students' participation in discussions.

\subsubsection{Designing Assessment that Connects to Pre-class and In-class Activities}

Teachers can also check students' preparation before class by building a strong connection between pre-class exposure, in-class activities, and assessments. Two courses connected students' preparation for class to students' performance in in-class activities, which was part of the assessment. In the first course, students were required to watch a 10-15 minutes video clip on teaching and write a lesson plan on the clip with possible ways of improvement as pre-class exposure. In the class, students discussed each other's plan, presented their findings, modified their plan based on their findings, and performed trial teaching. They were required to design a worksheet after the class. All tasks above would contribute to their final grade. In another course, students were required to read assigned text, search additional sources from the web, and have a showroom visit as pre-class exposure. In the class, students worked in groups to brainstorm and present a tactical marketing plan on improving the showroom they had visited. This presentation was worth $20 \%$ of the final grade. These two assessment designs allowed the teachers to observe students' preparation through their performance in the in-class activities, which is weighted in their final grade. In addition, it is believed that students can achieve learning goal easier if pre-class exposure, in-class activities, and assessment are strongly connected (Ginns and Ellis, 2007). 


\subsection{Activities}

\subsubsection{Reducing the Types of In-class Activities}

As stated above, assigning too many types of in-class activity poses a challenge to the flipped classroom. Reducing the types of in-class activities can "allow students to become familiar with the active learning strategy and avoid the risk of students focusing on the process of the strategy rather than the learning related to content" (Gilboy et al., 2015, p. 112). Most of our teachers chose to reduce the types of in-class activities by implementing flipped classroom partially. Among twenty-six courses, sixteen decided to have only 1 to 2 flipped classes, and eight planned for 3 to 6 flipped classes. The three courses that decided to flip all teaching classes (12 to 13) limited their types of in-class activities to group work, discussion and presentation. The limited types of in-class activities can help students focus on the content of learning.

\subsubsection{Designing Activities to Focus on Application and Practice}

In some courses, experiment or application of knowledge to cases formed the core of in-class activities. A marketing course had students' presentation of marketing strategies as its in-class activity. A course on science centered its in-class activity on conducting experiments. A course on nursing adopted simulation of a real-life situation in class. A course on electronic design required students to design electronic gadgets in class. Students were well aware of what they were expected to do in class. They were also well aware of the relevancy between the activity and the course, for they knew that in-class activity was an application of the theoretical knowledge taught in the course. Therefore, it was easier for them to navigate through the course. In addition, students can consolidate their knowledge and integrate what they learned into real life through these activities.

\subsubsection{Planning Interactive and Engaging Activities}

As stated, increasing interaction is an important advantage of the flipped classroom approach. Interaction with students is also a way to catch students' attention to in-class activities. Most courses in the project adopted activities that encourage interaction between teacher and student and among students into their classroom. Group discussion, presentation and group project were three common examples. Various software requiring individual responses from students such as uReply and clickers were introduced as additional measures.

One course encouraged interaction by introducing team competition into class. It employed different gaming software including Bingo, Kahoot!, Badaboom, and constructed a real-time board game with iPad for its in-class activity. The teacher of this course stated explicitly in the teaching profile that his class objective was to get students active in class and the characteristic of his flipped class was an engaging and fun classroom.

\subsubsection{Arranging After-class Activities}

In addition, some teachers planned after-class activities to further consolidate their students' knowledge. In one course on science, planting on a rooftop was employed as an after-class activity. Some courses required their students to write a short summary for in-class discussions or do calculation exercises after class. Other after-class activities included movie-watching, further discussions, post-class quiz, or teachers providing feedback to students.

\section{CONCLUSION}

This article examines the challenges of flipped classroom as demonstrated in previous literature through a framework according to the four key elements suggested by Brame. The four key elements are exposure, incentive, assess, and activities. Exposure is the materials provided for students to study before class. The quality of videos as exposure and students' need for assistance outside classroom pose a challenge to it. Incentives are provided for students to prepare for class. A score is a strong extrinsic incentive. The intrinsic incentive is on students' acceptance of the flipped classroom pedagogy. Challenges occur when students do not accept the pedagogy. Mechanism to assess students' understanding should be able to ensure students' 
preparation, but teachers find it difficult to do so. Activities in class should be designed to deepen students' understanding, enhance their skill, and promote interaction. However, the number of types of activities and the ability to engage students into activities pose challenges to the flipped classroom implementation.

Observing from the teaching profiles of the twenty-six teachers, this article suggests eleven tips on flipped classroom design that would be able to meet these challenges. Regarding exposure, (1) the quality of videos can be ensured by adopting existing videos, especially those with proven quality, and a variety of media can also be used as pre-class exposure. Besides, (2) additional assistance such as guiding questions and online discussion support can help students' preparation. For incentive, (3) scoring pre-class tasks is a strong extrinsic incentive for students, and (4) teachers can facilitate students' intrinsic incentive by addressing the advantages and benefits of the flipped classroom pedagogy. In assessing students' preparation, three kinds of assessment can help teachers ensure that students are well prepared: (5) assign pre-class tasks that cover pre-class exposure, (6) check students' preparation by in-class activities, and (7) design assignments that connect with pre-class and in-class activities. Regarding activity planning, (8) teachers should reduce the types of activities by sticking to a few types or implementing flipped classroom partially. (9) In-class activities can be designed to focus on experiments or application of knowledge. (10) Interaction and student's engagement can be increased by different kinds of activities, and finally, (11) some extra activities can be arranged after class to further consolidate students' knowledge.

The preliminary research has obvious limitations. Firstly, the tips provided were concluded from an observation of course design only. Their usefulness must be examined through observing the execution of those courses, which is also the focus in the next phase of our project. Future research assessing the effectiveness of the tips shall collect and examine two sets of data, including teachers' reflection on implementing their courses and students' opinion towards the flipped class they attended. Second, as mentioned in the introduction, teachers' willingness to participate, school support and technological support are also vital in the implementation of the flipped classroom. Due to the scope of this article, the challenges in these aspects were not assessed thoroughly. Further investigation in this research project would shed light on these aspects.

\section{ACKNOWLEDGMENT}

The authors would like to thank Ms. Ivy Lu Ming, Ms. Man Tsang Yuen Man, and Mr. Bernado Wong Yuk Wang from the Chinese University of Hong Kong; Dr. Crusher Wong and Ms. Angel Lu from City University of Hong Kong; Dr. Cheung Ka Lun and Ms. Rebecca Lin Xiaoying from the Education University of Hong Kong; Dr. Theresa Kwong and Dr. Isaac Chan Chi Fai from Hong Kong Baptist University; and Dr. Kevin Chan, Mr. George Cheung and Mr. Cypher Au Yeung from the Hong Kong Polytechnic University for their efforts in recruiting the teachers and collecting the teaching profiles. The authors also wish to thank the teachers for their contribution of completing the teaching profiles. This paper is part of the project "Effective Implementation of the Flipped Classroom Approach in Hong Kong Higher Education for Enhanced Learning Outcomes", which has been supported by the University Grants Committee of Hong Kong.

\section{REFERENCES}

Abeysekera, L. and Dawson, P. (2014). Motivation and cognitive load in the flipped classroom: definition, rational and a call for research. Higher Education Research \& Development, 34 (1), 1-14.

Akçayır, G. and Akçayır, M. (2018). The flipped classroom: a review of its advantages and challenges. Computer and Education, 126, 334-345.

Baker, J. W. (2000). The "classroom flip": using web course management tools to become the guide on the side. Selected Papers from the $11^{\text {th }}$ International Conference on College Teaching and Learning (pp. 9-17). Chambers, Jack ed. Jacksonville: Center for the Advancement of Teaching and Learning, Florida Community College.

Brame, C., (2013). "Flipping the classroom". Retrieved [7 Nov. 18] from http://cft.vanderbilt.edu/guides-subpages/flipping-the-classroom/.

Chen, L. et al. (2015). Students' perspectives of using cooperative learning in a flipped statistics classroom. Australasian Journal of Educational Technology, 31(6), 621-640. 
Chen, Y. et al. (2014). Is FLIP enough? Or should we use the FLIPPED model instead? Computers \& Education, 79, 16-27.

Fautch, J. M. (2015). The flipped classroom for teaching organic chemistry in small classes: Is it effective? Chemistry Education: Research and Practice, 16(1), 179-186.

Gannod, G. C. et al. (2008). Using the inverted classroom to teach software engineering. In ICSE '08: Proceedings of the 30th International Conference on Software Engineering (pp. 777-786). ACM, New York, NY.

Gilboy, M. B. et al. (2015). Enhancing student engagement using the flipped classroom. Journal of Nutrition Education and Behavior, 47(1), 109-114.

Ginns, P., and Ellis, R. (2007). Quality in blended learning: exploring the relationships between on-line and face-to-face teaching and learning. Internet and Higher Education, 10(1), 53-64.

Khanova, J. et al. (2015). Student experiences across multiple flipped courses in a single curriculum. Medical Education, 49(10), 1038-1048.

Koh, K. H. et al. (2018). Experience: Learner Analytics Data Quality for an eTextbook System. ACM Journal of Data and Information Quality, 9(2), 10:1-10:10.

Lage, M. J. et al. (2000). Inverting the classroom: a gateway to creating an inclusive learning environment. The Journal of Economic Education, 31(1), 30-43.

McGivney-Burelle and J., Xue, F. (2013). Flipping calculus. PRIMUS. 23 (5): 477-486.

Sams, A. (2011). There is no such thing as THE Flipped Class (5 OCT- 2011) http://chemicalsams.blogspot.com/2011/10/there-is-no-such-thing-as-flipped-class.html (retrieved on 3 Oct 2018)

Strayer, J. (2007). The Effects of the Classroom Flip on the Learning Environment: A Comparison of Learning Activity in a Traditional Classroom And a Flip Classroom that Used an Intelligent Tutoring System (Doctoral Dissertation). Retrieved from etd.ohiolink.edu/ (Accession No. osu1189523914)

Thai, N. T. T. et al. (2017). The impact of a flipped classroom design on learning performance in higher education: look for the best 'blend' of lectures and guiding questions with feedback. Computers \& Education, 107, 113-126.

Wanner, T., and Palmer, E. (2015). Personalising learning: exploring student and teacher perceptions about flexible learning and assessment in a flipped university course. Computers \& Education, 88, 354-369.

\section{APPENDIX: SAMPLE OF TEACHING PROFILE}

Flipped Classroom in Higher Education Project

Teaching Trials $\left(1^{\text {st }}\right.$ round; $1^{\text {st }}$ semester, 2018/19)

\section{$\underline{\text { Profile }}$}

\section{Course Information}

\begin{tabular}{|l|l|}
\hline Course name & \\
\hline Course code & \\
\hline Instructor name & \\
\hline Classroom & \\
\hline Lecture/ Tutorial (Time/Day) & \\
\hline Class size & \\
\hline
\end{tabular}




\section{Course Design}

Briefly illustrate how the flipped classroom approach is incorporated into the course design

\begin{tabular}{|l|l|}
\hline $\begin{array}{l}\text { Objectives of using the flipped } \\
\text { classroom approach in this course }\end{array}$ & \\
\hline $\begin{array}{l}\text { Number of classes that use the flipped } \\
\text { mode }\end{array}$ & \\
\hline Activities before class & \\
\hline $\begin{array}{l}\text { Ways to encourage students to } \\
\text { complete the above activities }\end{array}$ & \\
\hline Activities during class & \\
\hline Activities after class & \\
\hline Others & \\
\hline
\end{tabular}

